\title{
Calculation and Characteristic Research of Temperature Rise for Motor Temperature Field
}

\author{
Erbao $\mathrm{Lu}^{1, \mathrm{a}}$, Xiaorong Zhu ${ }^{1, \mathrm{~b}}$, Bin Du ${ }^{1, \mathrm{c}}$, Ye Deng ${ }^{1, \mathrm{~d}}$ and Chenghan $\mathrm{He} \mathrm{e}^{1, \mathrm{e}}$ \\ ${ }^{1}$ Beijing Research Institute of Precise Mechatronics and Controls, China

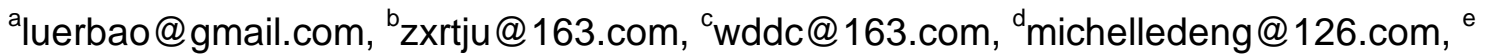 \\ hechh2@163.com
}

\begin{abstract}
Keywords:Temperature rise, Permanent magnet synchronous motor, Temperature field, Temperature rise characteristics

Abstract. Temperature rise caused by the loss of long-time working motor has negative effects on the working performance of the motor, as well as its service life and reliability. This article, analyses the calculation method of the motor's temperature rise based on the temperature field theory. On this basis, the temperature field and temperature rise characteristics of long-time working permanent magnet synchronous motor are studied. In addition, the defense measures are summarized to ensure the normal operation and performance of the motor.
\end{abstract}

\section{Introduction}

In the working process, temperature rise was caused by the loss of motor, which thrust an influence on the resistance value and magnetic linkage of the winding, thus to have negative effects on the working performance of the motor, and may cause damage or even burning accident of the motor's insulation layer [1,2]. For permanent magnet motor, temperature rise will exert an influence on the permanent magnet. For example, high temperature may cause magnet loss of permanent magnet [3,4]. Therefore, in a certain extent, temperature rise limits the improvement of the motor's performance, as well as its service life and reliability [5,6]. So temperature rise problem has become one of the key questions which has drawn widespread concern in the motor and its related field.

This article, based on the temperature field theory, analyses the calculation method of the motor's temperature rise. On this basis, the temperature field and temperature rise characteristics of long-time working permanent magnet synchronous motor were studied. Through analysis, we draw a conclusion that for the long-time working permanent magnet synchronous motor, temperature rise remains constant in the end, winding and the insulation parts of the motor possess the maximum temperature.

\section{Research of Temperature Rise Calculation Method Based on Temperature Field}

Currently, motor temperature rise calculation method mainly has three kinds: simplified formula method, equivalent heat circuit method, and temperature field method. The former two methods have flaw in computational accuracy. They can't easily calculate the temperature changes with time. As for the temperature field method, it solves the heat conduction equation making use of modern numerical methods, namely decomposing the solved region into many small units, establishing an equation for each unit, and then to solve the equation set as a whole. The temperature field method shifts its study object from macro perspective into micro, and from the whole into parts, thus to acquire the temperature and the rise of it on every point. Reliable calculating data of each unit of the whole calculation area are easily got, this makes the guidance of the motor design more accurate and reasonable.

\section{The Theory Base of Temperature Field Calculation}

Temperature field is the general name of the temperature distribution of all point of an object in a moment. In rectangular coordinate system, temperature field is the function of space coordinates and time, the function is expressed as follow: 


$$
T=f(x, y, z, t)
$$

In the equation above, $\mathrm{T}$ stands for the temperature of the object (unit: ${ }^{\circ} \mathrm{C}$ ), $x, y, z$ represent the three directions of the space coordinates in the rectangular coordinate system, and $t$ is time.

Eq. 1 shows the three-dimensional unsteady temperature field of an object, whose temperature changes in all the three directions of space and the time. According to the energy conservation law, the transient heat in the motor during the transient heat transfer process can be formulated as follow:

$$
[C]\left\{T^{\varphi}\right\}+[K]\{T\}=\{Q\}
$$

In this equation, $[K]$ is transfer matrix, it includes thermal conductivity, emissivity, convection coefficient and shape factor. $[C]$ is heat capacity matrix. $\{T\}$ stands for node temperature. $\left\{T^{\varphi}\right\}$ is the derivative of temperature to time. $\{Q\}$ is node heat flow rate vector containing heat generation.

In the three-dimensional Cartesian coordinates, the basic equation of the three-dimensional transient heat conduction is:

$$
K_{x} \frac{\partial^{2} x}{\partial x^{2}}+K_{y} \frac{\partial^{2} y}{\partial y^{2}}+K_{z} \frac{\partial^{2} z}{\partial z^{2}}+q=c \rho \frac{\partial T}{\partial t}
$$

In this formula, $\mathrm{T}$ is the temperature of motor, unit ${ }^{\circ} \mathrm{C} ; K_{x}, K_{y}, K_{z}$ are the thermal conductivity in $x, y, z$ directions of the motor's medium; $q$ is heat flux per unit time, unit $\mathrm{W} /\left(\mathrm{m}^{3}\right) ; c$ is specific heat capacity, unit $\mathrm{J} /\left(\mathrm{Kg} \cdot{ }^{\circ} \mathrm{C}\right) ; \rho$ is density, unit $\mathrm{Kg} / \mathrm{m}^{3}$.

\section{Boundary Conditions of Heat Conduction Differential Equation of Temperature Field}

The differential equation of heat conduction is built up according to the Fourier law and the law of conservation of energy, it's a relational expression which describes the temperature change with space. In order to solve the heat conduction differential equation, the boundary conditions of the object heat conduction differential equation should be given. Common boundary conditions can be divided into three kinds :

The first kind of boundary is the temperature of the object's boundary surface at any time, namely:

$$
\left.T\right|_{L_{1}}=T_{0}
$$

Where $L_{1}$ is the boundary of solving domain; $T_{0}$ is the temperature value of the steady state heat conduction process.

The second kind of boundary is heat flux density at the boundary of the object at any time, and the boundary conditions can be written as bellow:

$$
\left.\frac{\partial T}{\partial n}\right|_{L_{2}}=-\frac{q_{0}}{\lambda}
$$

Where $q_{0}$ is a given heat flux density which goes through the boundary surface of $L_{2}$.For the steady state conduction process, $q_{0}$ is a constant. As for the non-steady state process, $q_{0}$ changes with time. If the boundary surface is adiabatic, according to the Fourier law, Eq. 5 can be rewritten:

$$
\left.\frac{\partial T}{\partial n}\right|_{L_{3}}=0
$$

Eq. 6 indicates that the temperature change rate of $L_{3}$ boundary surface is 0 .

The third kind of boundary is the temperature of the fluid around the boundary, namely $T_{f}$, and the coefficient of heat transfer, namely $\alpha$, are known. According to the Newton's law of cooling, the convective heat transfer between the object surface $L_{3}$ and the fluid can be expressed bellow:

$$
q=\alpha\left(T-T_{f}\right)
$$


Where $T_{f}$ is the temperature of the object's boundary cooling medium.

On the basis of the Fourier law, the formula of the third boundary condition is:

$-\left.\lambda \frac{\partial T}{\partial n}\right|_{L_{3}}=\alpha\left(T-T_{f}\right)$

In this formula, $\alpha\left(\mathrm{w} / \mathrm{m}^{2} . \mathrm{k}\right)$ and $T_{f}$ can be constant, and they can also be a function that varies with time and position.

By the variation principle, the corresponding conditional variational problem of heat conduction differential Eq. 8 is:

$$
\left\{\begin{array}{l}
\left.F(T)=\frac{1}{2} \int_{V}\left[\lambda_{x}\left(\frac{\partial \mathrm{T}}{\partial \mathrm{x}}\right)^{2}+\lambda_{y}\left(\frac{\partial T}{\partial y}\right)^{2}+\lambda_{z}\left(\frac{\partial T}{\partial z}\right)^{2}\right)+\frac{c r}{2} T \frac{\partial T}{\partial t}-q T\right] d V+\frac{1}{2} \alpha \int_{s}\left(T-2 T_{f}\right) T d s=\min \\
S_{1}: T=T_{0}
\end{array}\right.
$$

Where $V$ is the solving domain.

The condition for the functional $F$ to get a extremum value is $\frac{\partial F}{\partial T}=0$. After discretization of the variational problem, a two dimensional temperature field finite element equations can be obtained:

$K \cdot T=F$

Where $T$ is an unknown node temperature column vector; $F$ is a load columns vector of node temperature $; K$ is coefficient matrix, known as the general temperature stiffness matrix.

Solving the above equation by the $L D L^{T}$ method, the temperature of all the nodes in the domain can be obtained. And all the nodes' temperature in the domain can be calculated by using the finite element software (such as ANSYS).

\section{Long-time Working Temperature Field Measurement Experiment and Temperature Rise Characteristic Analysis}

Fig. 1 is a permanent magnet synchronous motor $\left(P_{N}=1.5 \mathrm{~kW}, T_{N}=9 \mathrm{~N} . \mathrm{m}\right)$ in the condition of natural cooling. It works under the constant load of 5N.m. This figure shows a temperature field distribution map, in which the motor and the external heat exchange reach the equilibrium.

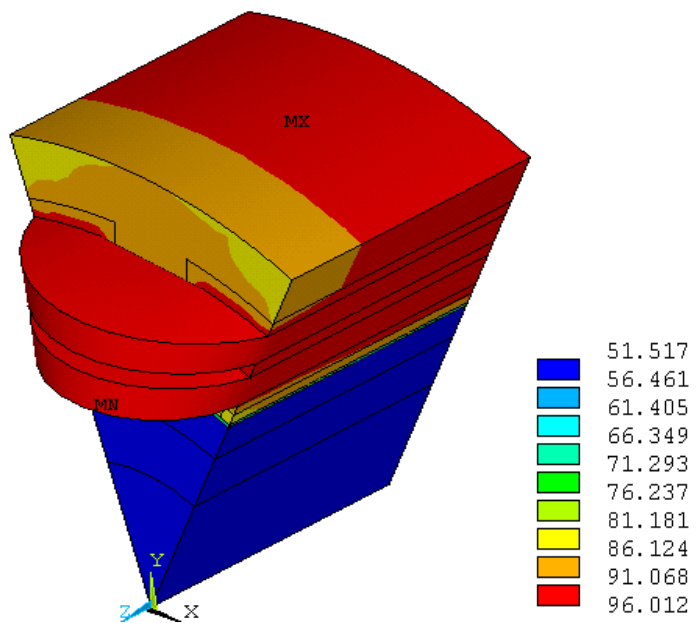

a) Temperature field distribution map of electric motor

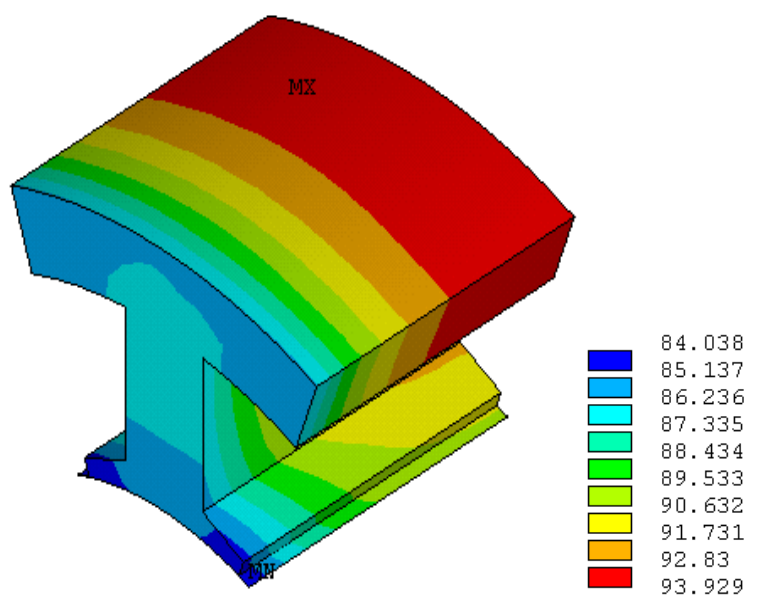

b) Temperature field distribution map on the motor's stator core

Fig.1 Temperature field distribution map (1/9 model) 
From Fig.1, it's not difficult to see that the temperature of the motor's copper conductor and its insulation are the maximum, and the stator core's temperature is just next to it. The axial center of the motor has the highest temperature, while the both ends of it are the lowest.

Fig. 2 shows the temperature rise curve of the motor's each parts. It can be seen from the curve that the temperature of each single part rise quickly at first, but when the motor reaches thermal equilibrium, temperature throughout the motor starts to keep unchanged. The temperature of conductor and insulation rise the fastest, and their stable temperature value is the highest, which is followed by the motor iron core teeth and yoke.

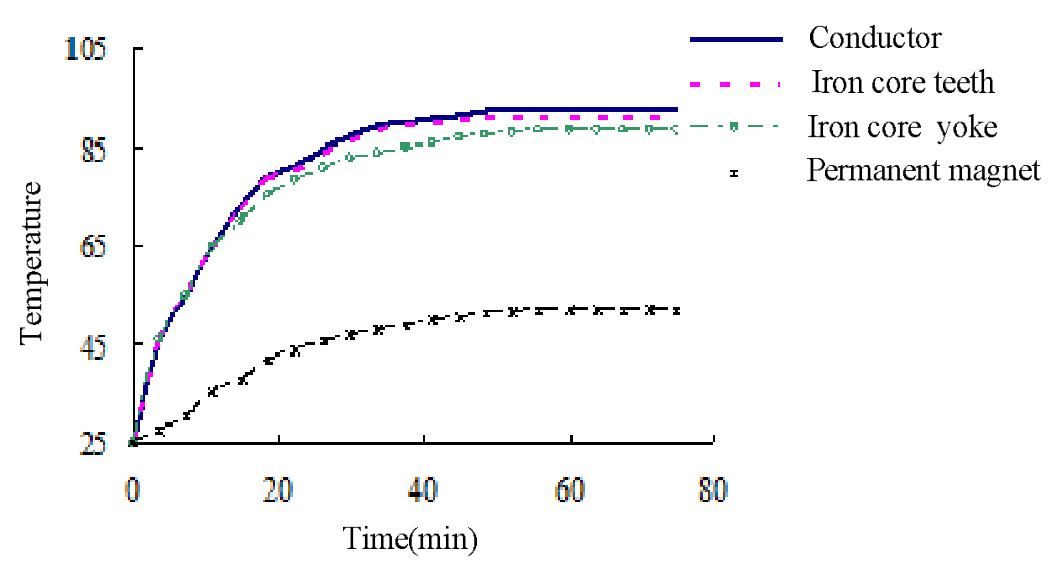

Fig.2 Temperature rise curve of motor's each part

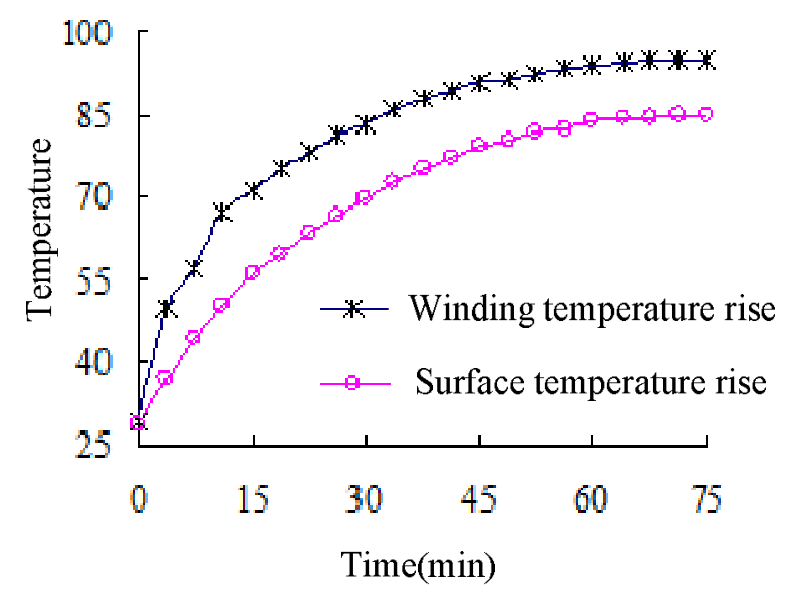

Fig.3 Measure the temperature rise curve of the motor by experiment

Finally, the motor temperature field and temperature rise calculation results are verified through experiments. The experimental curve is as shown in Fig.3.

The result above, which is based on temperature rise calculation and measurement of long-time working permanent magnet synchronous motor, further reflects the fact that, for reasonably designed motor, during continuous working process, its internal and external heat exchange activity will eventually reach a balance, and maintain in the safety temperature rise range at last.

The motor temperature rise rule above is basically suitable for all kinds of long-term and safety working motor. But for motors of different type and structure, due to the diversity of their characteristics like loss generation, ventilation and heat dissipation, and cooling methods, the motor's temperature rise rule should be correctly calculated and measured according to the specific circumstance of their own. 


\section{Conclusions}

Motor temperature rise is a complicated physical process which is closely related to the electromagnetic relationship of the motor. Therefore, in the motor research and application process, temperature rise of the motor should be calculated according to its specific characteristic. The calculation of the motor's temperature rise is based on the basic law of heat transfer, which is in firm tie with the calculation of the electromagnetic field. For long-time working motor, the temperature rise at each part of the motor finally stabilized at a constant value, and the winding and insulation have the maximum temperature. In order to prevent the motor from burning due to heat accumulation, on the basis of reasonable design of ventilation and cooling mechanism, thermal protection system is usually required.

\section{References}

[1] Ying H, Melnik R V N, Thogersen P B. Computational analysis of temperature rise phenomena in electric induction motors[J]. Applied Thermal Engineering, 2003, 23(7):779-795.

[2] Gnacinski P. Prediction of windings temperature rise in induction motors supplied with distorted voltage[J]. Energy Conversion \& Management, 2008, 49(4):707-717.

[3] Iwasaki N, Kitamura H, Kitamura M, et al. The Effect of Structure and Material Properties of Permanent Magnet Synchronous Motors on Temperature rise[J]. Journal of Vibration \& Control, 2015, 2010:61-66.

[4] Ruoho S, Kolehmainen J, Ikaheimo J, et al. Interdependence of Demagnetization, Loading, and Temperature Rise in a Permanent-Magnet Synchronous Motor[J]. IEEE Transactions on Magnetics, 2010, 46(3):949-953.

[5] Liu W, Zou H R, Tang S J, et al. Cooling Performance Analysis of the Permanent Magnet Motor[J]. Applied Mechanics \& Materials, 2015, 789-790:779-783.

[6]Zhang R, Wang H. Validation of a Temperature Rise Model of DC Permanent Magnet Motor[J]. Science \& Technology Review, 2010, 28(03):63-65. 